\title{
Point of Care Diagnostic Devices for Rapid Detection of Novel Coronavirus (SARS-nCoV19) Pandemic: A Review
}

\author{
Vamkudoth Koteswara Rao ${ }^{1,2 *}$ \\ ${ }^{1}$ Biochemical Sciences Division, CSIR-National Chemical Laboratory, Pune, India, ${ }^{2}$ Academy of Scientific and Innovative \\ Research (AcSIR), Anusandhan Bhawan, New Delhi, India
}

\section{OPEN ACCESS}

Edited by:

Yogendra Kumar Mishra, University of Southern Denmark,

Denmark

Reviewed by: Siddharth Sridhar,

The University of Hong Kong, Hong Kong

Suresh K. Verma, KIIT University, India

*Correspondence: Vamkudoth Koteswara Rao v.koteswara@ncl.res.in

Specialty section: This article was submitted to Biomedical Nanotechnology, a section of the journal Frontiers in Nanotechnology

Received: 11 August 2020 Accepted: 16 December 2020 Published: 02 February 2021

Citation: Koteswara Rao V (2021) Point of Care Diagnostic Devices for Rapid Detection of Novel Coronavirus (SARS-nCoV19)

Pandemic: A Review.

Front. Nanotechnol. 2:593619. doi: 10.3389/fnano.2020.593619
Coronaviruses are recognized as causative agents of human diseases worldwide. In Wuhan, China, an outbreak of Severe acute respiratory syndrome novel Coronavirus (SARS-nCoV-2) was reported at the end of December 2019, causing 63 million COVID cases and 1.3 million deaths globally by 2 December, 2020. The transmission risk forecasts and the SARS-nCoV-2 epidemic pattern are progressive. Unfortunately, there is no specific FDA approved drugs or vaccines available currently to treat SARS-nCoV-2. In response to nCoV-2 spread, the rapid detection is crucial for estimating the severity of the disease and treatment of patients. Currently, there are several RT-PCR based diagnostic kits available for SARS-nCoV-2 detection, which are time-consuming, expensive, need advanced equipment facilities and trained personnel. The cost of diagnosis and the unavailability of sufficient test kits may prevent to check community transmission. Furthermore, expanding the testing facilities in asymptomatic cases in hotspots require more Point of Care (PoC) devices. Therefore, fast, inexpensive, and reliable methods of detection of SARS-nCoV-2 virus infection in humans is urgently required. The rapid and easy-to-use devices will facilitate onsite testing. In this review, nucleic acid assays, serological assays, multiplex assays, and PoC devices are discussed to understand various diagnostic approaches to reduce the spread and mortality rate in the future. Aptamer based detection is most specific, inexpensive and rapid detection of SARS-nCoV-2 without laboratory tools. To the best of our knowledge more than 900 SARS-nCoV-2 test kits are in pipeline, among 395 test kits are molecular bested test kits and only few test kits are developed using Aptamer technology https://www.finddx.org/covid-19/pipeline/.

Keywords: SARS-CoV-2, aptamer, point of care, nanobiotechnlogy, biomedical application

\section{INTRODUCTION}

Viruses are infectious sub-microscopic agents, infecting humans, and animals (Adamczyk et al., 1999). About 6,000 species of viruses have been identified in the ecosystem in 1898 showing several biological activities (Virus Taxonomy, 2019). The smallpox virus was the deadliest and most devastating viral infection in the Indian agricultural communities about 11,000 years ago, and has been a pandemic in history (Ryan and Ray, 2004). In addition, about 500 million individuals were infected with the Influenza pandemic caused by the H1N1 virus in 1918-19. The mortality rate was very high at 100 million, and is the most calamitous in human pandemic disease history (Johnson and Mueller, 2002). 
Severe acute respiratory syndrome (SARS), Middle East respiratory syndrome (MERS), Coronaviruses (COVID-19) are known causative agents of diseases for various species of animals across the globe. However, diseases caused by coronaviruses and their symptoms vary from species to species. The highly publicized human coronavirus was discovered in 2003, inducing unique symptoms of SARS such as infections of the lower and upper respiratory tract (LRT/URT) (Burrel et al., 2020).

Unlike other viruses, SARS-CoV-2 has much greater global spread, infecting more individuals than combined infections of MERS and SARS. SARS-CoV-2 are enveloped positive strand (+) SARS-CoV-2 belongs to Coronaviridae family. Seven human SARS-CoV virus are known, and four strains show moderate signs of a common cold. The SARS_CoV-2 viruses are similar to previous human coronaviruses $(\mathrm{HCoV})$, which are $229 \mathrm{E}$ and OC43, Tsang et al. (2013), Fouchier et al. (2004), Woo et al. (2005), and Hilgenfeld and Peiris (2013) (Cascella et al., 2020). Also, HCoV 229E and NL63 are closely related which are belongs to the genus alpha-coronavirus, however, HCoV OC43, HKU1 and SARS-CoV-2 belongs to genera beta-coronavirus. SARS and COVID-19 are respiratory illness caused by specific $\mathrm{HCoV}$, but they are caused by different specific coronavirus. SARS caused by caused by a coronavirus are called SARS-COV. Novel coronavirus (nCoV-2019) was reported in Wuhan, Hubei province in China, 2019 marking the beginning of the pandemic caused by this virus. The estimates of the transmission risk of the newly discovered COVID-19 and the epidemic trend are progressive. The world health organization (WHO) has declared the Covid-19 epidemic threat as a "very high" level at more than 63 million infected individuals globally and 1.4 million deaths recorded globally till 2 December, 2020. This highly contagious virus has spread around the globe including 220 countries and territories.

\section{Structure and Genome of Virus and Host Interaction}

Novel virus SARS-CoV-2 belongs to the family Coronaviridae, genus Betacoronavirus which induces coronavirus disease in humans (COVID-19). The SARS (SL) virus BatCoV-RaTG13 have high sequence similarity with SARS-CoV-2 of $96 \%$, and with other closely related to two bats derived SARS coronaviruses (batSL-CoVZC45 and bat-SL-CoVZXC21), having 88\% over all sequence similarity with SARS-COV (de Carvalho and Nogueira, 2020; Zhou et al., 2020). Bats are speculated to be reservoirs for various emerging viruses that cause infections to humans and animals. However, bats are the likely reservoirs or hosts for transmission CoVs.

SARS-CoV-2 belongs to enveloped viruses with a positivesense RNA (+ssRNA) genome of $27-34 \mathrm{~kb}$ in size, encoding 27 proteins. It is a large pleomorphic, spherical particle with a globular projection on the surface. The SARS-CoV-2 has 5'leader -UTR-replicase/transcript spikes (S), envelope (E), membranes $(\mathrm{M})$, nucleotide $(\mathrm{N})$-3poly 9A tail, all required to produce a structurally complete viral particle. The ORF 1a and lb occupy the first two-third of the genome, encoding the replicase/transcriptase which cleaves itself to form the nonstructural proteins (nsps) (Deckert et al., 2020). A large number of glycosylated spike proteins are highly conserved among HCoVs (S1 and S2) domains binds to the host cell receptor angiotensin-converting enzyme 2 (ACE2) that receptor recognition, cell entry into the host cells (Letko et al., 2020). The nucleocapsid $(\mathrm{N})$ protein is only one that binds to the RNA genome, and involved in the viral assembly and budding makes the nucleocapsid (Malik, 2020).

The infection route for COVID-19 is the respiratory tract. Hence, contact and aerosol transmission occur when in close contact with symptomatic/asymptomatic individuals. The mean incubation period is reported to be 5.1, 11.5 (97\% cases), and 14 days (1\%) of infection. The most infected cases are in the age group of 21-40 (Lauer et al., 2020). The relationship between age and incidence is not yet known as there is heterogenicity among the most infected age groups in the world. Among the covid-19 infected cases so far, probably more deadly to men than women, but scientists still aren't sure. One of the reasons could be the ACE2 receptors which have been known to play an important role in virus invasion of host cells (Bwire, 2020). These receptors are expressed at higher levels in males compared to females. Other factors such as smoking, which directly influence lung cells, diabetes, cardiovascular disorders, mostly found in males can also enhance the expression of the ACE2 receptors (El-Tholoth et al., 2020). Therefore, the rate of infection among men is greater than in women. It can lead to severe symptoms of respiratory system disease in some cases; otherwise, most of the cases have mild fever, cough, and tiredness. Initially, the infection shows flu-like symptoms but is symptom-less for few weeks in some cases. Scientists have cautioned that a mutation in the SARS-CoV-2 virus $\mathrm{S}$ protein called D614G makes the virus more contagious, infects cell lines in vitro more readily suggests higher viral loads in patients (Korber et al., 2020). An analysis of 82 virus strains in India showed that nearly $50 \%$ of them have the new mutation, while nearly two-thirds of strains have displayed this mutation globally. Therefore, it has become important that the diagnostic measures are sufficiently accurate to detect infection even in the presence of a very low concentration of viruses in blood samples.

\section{In vitro Diagnosis of SARS-CoV-2 Virus Infection}

To contain this viral pandemic; it is crucial to predict the extent of the disease for better patient care. RT-PCR based SARS-CoV-2 detection methods currently being used, are gold standards for quantitative detection, and time-consuming, expensive, and requires advanced equipment's, biosafety facilities, and welltrained personnel. The indigenous RT-PCR diagnostic kits in India have been developed by Mylab, India, whereas Roche Diagnostics is among the first to obtain the FDA approval for its diagnostic test kits using RT-PCR. Massive detection and rapid, fast, inexpensive diagnosis are imperative to overcome the current pandemic problem. Due to lack of affordable, easy to use diagnostic kits; the danger of community transmission lurks. Also, expanding the testing strategy for COVID-19 in asymptomatic cases at the hotspot requires large scale testing 
which is not possible with RT-PCR based testing. Here in this review, we discuss some alternative solutions for the testing of COVID-19.

\section{Aptamers Based Diagnosis of COVID-19}

Aptamers are third-generation molecular probes made of the short oligonucleotide, with specificity and affinity toward the target molecule. Aptamers have lower molecular weight and size $(<10 \mathrm{kDa})$ than the antibodies and can bind efficiently with the target. The synthetic material is prepared through the systematic evolution of ligands by exponential enrichment (SELEX). Up to now, several SELEX methods such as capture-SELEX, immuno-SELEX, high-affinity resins, capillary SELEX, magnetic bead based SELEX etc. have been used to detect specific targets. Several billion copies of the chosen aptamer can be produced, at a time. Moreover, the specificity and sensitivity of aptamers are in par with any given antibodies. Aptamers have many more advantages than antibodies. These probes are easy to generate in the laboratory within a limited period, very cost-effective when compared to the current probes such as antibodies and sensors. Currently, no commercial solutions have been developed, for COVID-19 testing based on aptamer. Hence, aptamer-based kits open up new diagnosis solutions for the rapid and robust detection of SARS-CoV-2 viruses in human samples.

The workflow is very simple and defined. Aptamer assay is used to detect the target protein, peptide, small molecules, amino acids, nucleotides, antibiotics etc., which is as an alternative to fluorescence-based assays (Shafiei et al., 2020). Briefly, the specific target proteins and ssDNA oligonucleotides library are packed and incubated in the Ni-NTA column and repeatedly washed with a washing buffer to achieve high binding efficiency. Consequently, unbound oligonucleotides are eliminated by repeated washing. The bound oligonucleotides are then eluted and used as a template for PCR reaction. The final pool is cloned and expressed in a suitable vector. The obtained clones are evaluated using colony PCR, and positive clones with enhanced target specificity, are sequenced and secondary structures, are predicted using M-fold software tools. The binding efficiency of the aptamer target proteins can be evaluated with the help of FRET/SPR/RT-PCR. These aptamers can be used in conjugation with fluorescence and other assays to develop rapid, low-cost, sensitive, easy-to-use COVID-19 PoC tools, which can be useful for non-experts (Bai et al., 2018; Samson et al., 2020).

APT diagnostics (AptaDx) is a world-leading company working on nucleic acid aptamer selection having different platforms including, Aptamer solutions and Aptamer therapeutics and Aptamer diagnostic products (https://www. aptamergroup.co.uk/about-us/). Their diagnostic platform can be used to develop COVID-19 PoC diagnostic kits. Using similar approaches/techniques rapid microbiology has developed "Pinpoint's Low-Cost Handheld COVID-19 Aptamer-based Diagnostic Device" which is an accurate COVID-19 diagnostic test that can be executed by non-clinicians (Rapid Microbiology, 2020). Translational Health Service and Technology institution
(THSTI, India) developed DNA aptamer linked immunosorbent assay for rapid detection of novel coronavirus with $90 \%$ selectivity and $97 \%$ specificity. To the best of our knowledge more than 900 SARS-nCoV-2 test kits are in pipeline, among 395 test kits are molecular bested test kits and only few test kits are developed using Apatamer technology https://www.finddx.org/covid-19/ pipeline/.

The detailed PoC diagnosis, serological and multiplex system based diagnostic tools are summarized in Figure 1. In the aptamer-based diagnosis of COVID-19, samples lyzed with cell lysis solution can be directly processed for the diagnosis; RNA isolation, a typical step in the other diagnosis solution for COVID-19 is not required here. These extremely selective bioligands are capable of binding in the range of picomolar $(\mathrm{pm})$ to nanomolar ( $\mathrm{nm})$ concentration (10-12-10-9). Also, infected individuals can reach a good number of viral load within 1-3 days of the infection for testing target proteins to detect viral infection (Chen et al., 2020c). Aptamer based assay can be generated using multiple technologies. Most of the DNA, RNA, and peptide-based assays can be converted to aptamerbased assay for the detection of viral proteins in general and SARS-CoV-2 virus in specific, with higher efficiency. Here we discuss some of the technologies which are/and can be conjugated with aptamers to develop a diagnostic assay for COVID-19 detection.

\section{Aptamer-Chemiluminescence Immunosorbent Assay}

Aptamer immunosorbent chemiluminescence essay is similar to other immunoassays such as Radioimmunoassay (RIA), Fluorescent Immunoassays (FIA), Enzyme-linked immunosorbent assay (ELISA) that combine chemiluminescence technique with immunochemical reactions. Such aptamer-based assays have been reported for the detection of $\mathrm{HBV}$ infection (Jacobs et al., 2018; Ha et al., 2020). The difference is the presence of aptamer coating, instead of a specific antibody. These short sequences of RNA oligonucleotides are unique for the target viral proteins (Jin et al., 2020). Initially, aptamer and viral protein bind together, later enzyme-conjugated antibody and luminescence substrate are added for viral protein detection. The aptamer-ab hybrid immuno-chemiluminescence assay can detect viral protein at a very low concentration $(2 \mathrm{pg} / \mathrm{ml})$. Such RNA-aptamers are new kinds of biosensors with high binding affinity and target specificity. Methods of aptamerchemiluminescence show a wide range of advantages, have a simplified handling process and are attracting increased attention.

\section{Magnetic Chemiluminescence Enzyme Immunoassay}

In this method, viral protein-encoding specific peptides are used for the detection of IgM and IgG antibodies against SARS-CoV-2 (Cai et al., 2020). These synthetic peptides are conjugated with bovine serum albumin (BSA) by amino or carboxyl-terminal cysteine and biotin. Each biotin-labeled peptide is bound to 


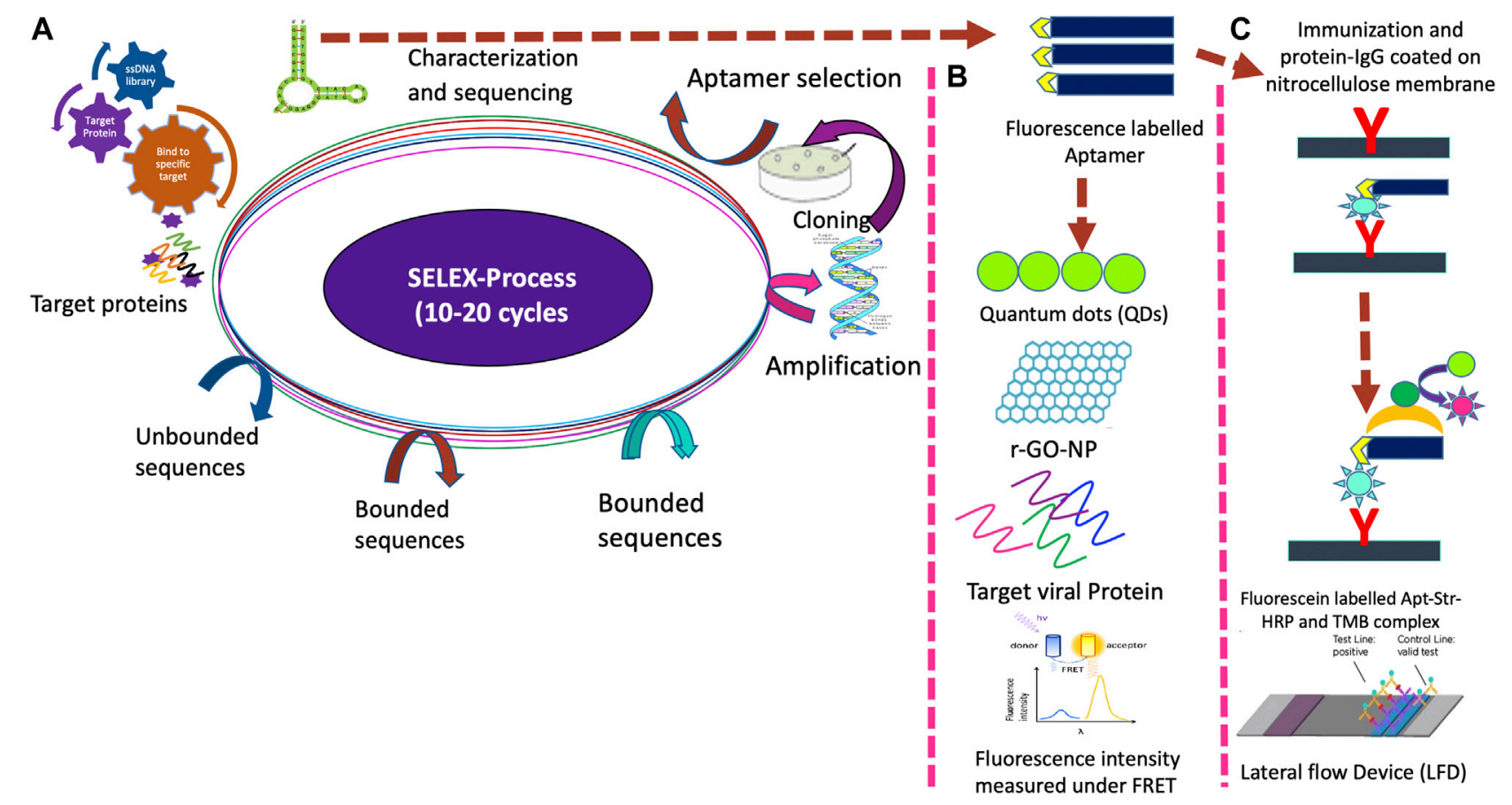

FIGURE 1 | Novel ssDNA/RNA aptamer biosensing platform for detection of novel coronavirus (SARS-CoV-2) using nano-biotechnology approaches. (A). Development of aptamer using the SELEX process. (B). Aptasensors for the multiplex analysis. (C). Lateral flow device.

streptavidin-coated magnetic beads. When a patient's serum is combined with beads, the antibodies recognize viral peptide present on the magnetic beads. The conjugated $\mathrm{Ab}$ and the luminescence substrate were added to the beads to detect complexes of $\mathrm{Ag}-\mathrm{Ab}$ using a luminescence reader. This approach, being very precise showed 71.4 and $57.2 \%$ accuracy against SARS-CoV-2 virus infection using IgG and $\operatorname{IgM}$, respectively. IgG could be identified at an early stage of infection just after two days, but IgM could be detected only at later stages of infection. These chemiluminescence-based techniques are very sensitive and can be performed within a few hours. If the result is negative for $\operatorname{IgG} / \operatorname{IgM}$ testing, the chemiluminescence assay could be followed by RT-PCR testing to increase the accuracy of the COVID-19 diagnosis. This method has the advantage of detecting viral proteins present in the sera rather than RNA. RNA isolation is a limiting step as it requires $3-4 \mathrm{~h}$, skilled personnel, and specialized instruments. As chemiluminescence readers are portable, this assay could be developed into a Point of Care (PoC) diagnostic kit.

\section{Surface Plasmon Resonance}

Surface Plasmon Resonance (SPR) is a highly sensitive technique to detect the virus by measuring the binding affinity of an aptamer to viral proteins by immobilization on SPR chips, which results in the metals' free electrons resonance (Zou et al., 2019). When an aptamer conjugates with the gold nanoparticles (AuNP), the virus protein binds to the Apt-AuNP complex and brings about a change in the mass and results in a refractive index shift which can be quantified by intensity of the polarized light (Kumar et al.,
2018). Avian influenza virus (H5N1), Human Immuno virus (HIV-1) tat protein are also identified using SPR-aptasensors (Lawrence et al., 2009; Rahim Ruslinda et al., 2013). As it can be automated, it outshines other RT-PCR alternatives, requires few biological reagents, and generates results in a few minutes. This assay also overcomes the difficulties of working with RNA as it is based on viral protein detection. The downside of this approach is that the equipment is expensive, hence this assay cannot be developed into a Point of Care (PoC) diagnostic kit.

\section{Aptamer Based Fluorescence}

Fluorescence resonance energy transfer (FRET) is a physical phenomenon, which is being used more and more in biomedical research and drug discovery today. FRET relies on the distance-dependent transfer of energy from a donor molecule (chromophore) to an acceptor molecule. The transfer of energy leads to a reduction in the donor's fluorescence intensity, and the excited-state lifetime, and an increase in the acceptor's emission intensity. The interactions between ssDNA/RNA, nanomaterial, and the target are combined to develop a sensitive and selective method for a fluorescence quenching detection platform. In rGOSELEX, the reduced graphene oxide (GO) interacts with the nucleotides and helps in the selection process. This is an immobilization-free process and hence consumes less time. Whereas, the capture-SELEX involves immobilization of the nucleotide sequences leading to the specific selection of the ssDNA against the target. The aptamer is connected to a fluorophore and quencher is used to quench its signal by FRET. Graphene is widely used as a quencher since it is an 
energy acceptor. The fluorescence signal intensity varies as aptamer and target interact which can be used to define and measure the target concentration. The developed novel aptamer is labeled either with biotin or fluorescein amidite (FAM) for multiplex assays using FRET detection (Lee et al., 2017). The biotin-labeled aptamer is used for the multiplex detection of the target, using Förster resonance energy transfer (FRET) spectroscopy as binding induces a conformational change altering the distance between the reporter molecules. Briefly, a complementary oligonucleotide labeled with a quencher can hybridize with aptamer which switches off fluorescences. After binding with the target, the conformational shift in the aptamer results in elution of the complementary oligonucleotide turning on fluorescence in a quick, quantitative gain of signal (Loeffelholz and Tang, 2020). Since handheld readers are available to measure the fluorescence, this assay has the potential to be developed into a Point of Care (PoC) diagnostic solution.

\section{Aptasensors-Raman Spectroscopy}

Raman spectroscopy can be complementary to RT-PCR-SARSCoV-2 detection, and even its mutants. Aptamers can be used to detect specific molecular diagnostic markers (Lu et al., 2020). Highly specific Raman spectroscopy can be used for the analysis of biofluid samples directly, to measure the virus-binding efficiency of ACE2 receptors of the host cells (Neuman et al., 2008). Newsfile Corp. BioMark Diagnostics Inc. has developed a novel non-invasive Raman probe to be used in diagnostics for cancer and certain other pathogens (https://www.newsfilecorp. com/release/53443/BioMark-Diagnostics-Advanced-RamanSpectrometer-Could-Potentially-Serve-as-ComplementaryDetection-Tool-for-COVID-19-Coronavirus). It is simple, rapid, versatile, high throughput, and portable. A single virus can be trapped in a focused laser beam and can be studied for its characteristics. Once the tip is positioned in the laser spot, the sample is moved under the tip and the region of interest is selected. After setting a profile of grid lines on the sample, the AFM (Atomic Force Microscopy) is coupled, to the Raman spectrometer for obtaining enhanced Raman spectra. The scientific goal, in addition to the identification of virus, is to identify structural changes of the virus surface at an early stage, using Tip-Enhanced Raman scattering (TERS). In contrast to PCR-related techniques, in TERS a separation of the different components or labeling is not required, and ultimately a single virion is sufficient for identification. Due to surface specificity, it allows early detection of change that alters the antigenic properties of viruses and, thus the effectiveness of vaccines, with the smallest sample quantities (Deckert et al., 2020).

\section{Nanoarray Aptamer Chip Assay}

Nanoarray aptamer chip assay is a nano-sized system for the detection of viral infections (Ahn et al., 2009). This technique uses dip-pen nanolithography in which an atomic force microscope (AFM) tip is used to draw patterns on the surface of a glass plate. A specific aptamer is then coated on the surface later in the patterns. As this is a nano-based process, viral protein detection only requires $10 \mu \mathrm{l}$ of the samples. Once the complex of aptamer and viral protein is formed, antibodies are added to it. This Ag-Ab complex is detected using an anti-antibody labeled with the fluorescence probe and observed under the fluorescent microscope (Zon, 2020). Being a nanodevice, this technique is very sensitive and a very small sample volume is needed, but it requires a sophisticated instrument for assay, which is its main drawback.

\section{Proteome Microarray}

The preliminary approach till now has been to detect the viral infection using nucleic acid as a diagnostic measure. Specific SARS-CoV-2 structural proteins and ORF are the most significant parts of the virus that elicit an immune response and are useful in the development of specific diagnostic tools (Chen et al., 2020a). The peptide library of 15 amino acid long and 5 amino acid overlap is generated, using synthetic genes. The peptides are labeled at the C-terminal along with full-length target protein sequences and coated on a microscope slide using biotin-streptavidin for fluorescence signals. A serum sample is applied to the slide and incubated to test the COVID-19 virus infection. Viral protein-specific antibodies bind to the peptides, and conjugated anti-SARS antibodies are applied to detect this complex, as performed in the other diagnostic processes. This method can be performed within 90 min rather than 3-4 h for $\mathrm{RT}$-PCR reaction with a sensitivity of $94 \mathrm{pg} / \mathrm{ml}$ sample. Using the same techniques, IgG and IgA epitope profiling was performed and it was found that about $80 \%$ of antibodies produced in COVID-19 active patient serum are specific for $\mathrm{N}$ and $\mathrm{S}$ protein (Jiang et al., 2020).

\section{Nucleic Acid-Based Molecular Diagnosis of COVID-19}

The polymerase chain reaction (PCR) is the basic identification technique using their genetic materials. Molecular based detection of SARS-CoV-2 includes the reaction mixture consisting of +ssRNA genomic regions of ORF1b/ORF8 and the structural proteins; and RNA-dependent-RNA polymerase (RdRP) are widely used for the diagnosis (Esbin et al., 2020).

\section{Real-Time Polymerase Chain Reaction/ Reverse Transcriptase (RT-PCR)}

Currently, RT-PCR is the gold standard for SARS-CoV-2 virus detection, and it amplifies RNA even in very low concentrations. RNA in the patient's sample is converted into cDNA by RNA dependent DNA polymerase and then PCR amplification was performed with two specific primers of SARS-CoV-2 virus. If RNA is detected in the sample of the patient, it is positive for COVID-1,9, and in rare cases, this molecular test may give falsenegative results if viral RNA is too low (Lee, 2020).

Since coronavirus has ssRNA as genetic material, the use of RT-PCR for viral detection is favored over the normal PCR as it reduces the conversion step of RNA into CDNA, which must be performed separately in normal PCR. The two-step PCR is timeconsuming, as opposed to one-step PCR, and is laborious (Smyrlaki et al., 2020). 
Instead of two steps RT-PCR process, several firms have developed one-step RT-PCR in a single tube. Mylab, India developed RT-PCR based COVID-19 diagnostic test kits (RTPCR), approved by FDA/CDSCO for emergency use authorization (EUA). The RT-PCR technique is widely used to detect the SARS-CoV-2 virus. It is very effective and diagnoses infection at an early stage, but it is a very timeconsuming process. The high mutation potential of the virus due to ssRNA as genetic material makes this technique not use a for long time without new primers and optimization. RTPCR COVID-19 test kits have many disadvantages. They are time-consuming, need transportation of samples, require sophisticated Instruments, laboratory setting, up and trained personnel.

\section{Sensitive Splint-Based One-Step Isothermal RNA Detection}

SENSR method is used to identify viruses rapidly. The onsite process consists of a ligation reaction and subsequent transcription by T7 RNA polymerase. The clinical sample is added in a reaction mixture comprising of oligonucleotides, probes, RNA template, SplintR ligase, T7 RNA polymerases, and fluorogenic dye (Woo et al., 2020). The reaction mixture is incubated followed by termination by heating at $95^{\circ} \mathrm{C}$. The DNA probes are ligated to the target RNA, present in the sample to form RNA aptamer, which is then transcribed by T7 RNA polymerase. The fluorogenic dye binds to a specific site on this product, and this helps detect virus infection. This method is fast, sensitive and doesn't require large expensive equipment, hence it has the potential to be developed into a point of care $(\mathrm{PoC})$ diagnostic solution.

\section{Closed-Tube Penn-RAMP}

Loop-mediated isothermal amplification (LAMP) is a single tube DNA amplification technique; an alternative to detecting SARS-CoV-2 and certain other viral diseases (Ren et al., 2020). Reverse transcription loop-mediated isothermal amplification (RT-LAMP) combines LAMP with a reverse transcription step and allows for RNA detection. To maximize specificity; conserved SARS-CoV-2 sequences were used to generate LAMP primers (Rosenwald., 2020). This test can be performed to diagnose SARS-CoV-2 infection in a close tube. A nasal swab is added into the tube, and then a mixture of RPA and LAMP are added in the ratio of 1:9, and the reaction is performed in a thermocycler at $38{ }^{\circ} \mathrm{C}$ for initiation of recombinase polymerase Amplification (RPA) reaction. It is then incubated at $63^{\circ} \mathrm{C}$ for completion of the second stage. The deep violet color, with the addition of chromogenic reagent (leucocrystal violet dye), indicates positive for COVID-19. This technique is more sensitive than RT-PCR or LAMP alone (Kim et al., 2020). With a single closed tube test with colorimetric readout, this test is an ideal candidate to be developed into the Point of Care diagnostic kit.

\section{Other Molecular Diagnostic Methods for COVID-19 Detection}

A new device, Abbott ID Now COVID-19 test-launched in the United States (US) for the detection of COVID-19 has been approved by FDA on emergency use authentication (EUA) which delivers fastest results in as little as $5 \mathrm{~min}$ (Abbott, 2020; ID NOW $^{\mathrm{TM}}$ COVID-19, 2020). Interestingly, CRISPR-based SHERLOCK (Specific High Sensitivity Enzymatic Reporter UnLOCKing) is also being used for the detection of SARSCoV-2 virus infection in humans. The RT-PCR based diagnosis, rolling circle amplification, nucleic acid hybridization, metagenomic sequencing, lateral flow immunoassay for detection of specific antibodies, LMAP, RPA techniques are currently available molecular methods for diagnosis of SARS-CoV-2 infection in humans.

\section{Immunological/Serological Diagnosis of COVID-19}

The immune system develops an antibody to the virus within a few days, which could be detected. Detection of an antibody is usually achieved using Enzyme-linked immunosorbent assay (ELISA). ELISA is used to identify and measure specific peptides, proteins, and antibodies (Ab). Recombinant polyclonal/monoclonal antibodies $(\mathrm{pcAb} / \mathrm{mAb})$ are generated against viral structural protein by injecting viral recombinant structural protein. Such recombinant polyclonal antibodies (pcAb) are coated on the wall of the 96-well plate. Conjugated anti-Ab is applied to the plates; the substrate is added, and the development of color indicates positive for the virus infection (Shanmugaraj et al., 2020). The viral protein can be detected from day 6-24 in nasopharyngeal aspirate, 8-32 days in the fecal sample, and 11-31 days in a urine sample (Sergei et al., 2020). Therefore, this technique is more sensitive, rapid, and simple as compared to other complicated techniques in the detection of SARS-CoV-2 viral infection. The inability to detect early stage infection in fecal and urine samples is one of the drawbacks of this method, and sometimes cross-reactivity is another issue.

\section{Other Diagnostic Methods Mass Spectrometry Analysis}

It is an analytical technique that measures the mass-to-charge ratio of the samples generated by ions, and the resulting output is in the form of the mass spectrum (Nikolaev et al., 2020). This method is useful in molecular recognition and detection of the chemical components. Genes for the viral protein, are first cloned and expressed into an appropriate vector, purified proteins are digested using trypsin and then, are loaded onto the capillary column of silica. Then the entire column is positioned inside the quaternary high-pressure liquid chromatography which is used to isolate various molecules in a sample. The peptides/proteins are eluted from the column after running the sample with appropriate buffers, and they are directly electrosprayed into ion trap mass spectrometer, and the MS spectra of nonstructural SARS-CoV 2 proteins are used to analyze the patient's samples (Ihling et al., 2020). 


\section{The Matrix-Assisted Laser Desorption Ionization Time-of-Flight Mass Spectrometry}

The MALDI-TOF mass spectrometry is a highly advanced method of protein pattern recognition to diagnose various diseases and health disorders (Rocca et al., 2020). MALDITOF is a preferable method because of low technical user requirements and minimal consumables. It uses digital data, analyzed by AI algorithms, and requires a small amount of blood, urine, or sputum sample. The analysis is data-rich; delivers test results within minutes and is economical. The sample can be re-interrogated with full scanning of all test panels from a single sample. This technique uses mass spectrometry to spot proteins that are unique to the SARSCoV-2 virus and deliver results within $30 \mathrm{~min}$. It can detect the presence or absence of COVID-19, measure the viral load, and detect new viral strains.

Current diagnosis relies primarily on PCR to determine the virus, but unique biomarkers need to be identified, to enhance the treatment approaches. For clinical studies, metabolomics is primarily important, as it involves the physiological pathways that are altered, in response to the disease. The initial goal of COVID-19 research based on metabolomics should be to identify biomarkers, which facilitate stratification or enable clinicians to access or predict therapeutic response. Metabolomic approaches such as gas chromatography-mass spectrometry (GC-MS), liquid chromatography-mass spectrometry (LC-MS), nuclear magnetic resonance (NMR), and capillary electrophoresis-mass spectrometry (CE-MS) are being defined (Fraser et al., 2020). Comparing metabolomics between non-severe and severe groups of patients for determining the changes in the host metabolite profile would be a novel approach and help to find new goals for therapy by defining possible markers to differentiate the patients. Depending on biomarker determination, one can easily differentiate between moderate and extreme cases of COVID-19 from detailed metabolomic investigations. Complete metabolome or proteome analysis of COVID-19 patient's clinical samples is needed to indicate the severity of the novel COVID-19. It is a clear form of metabolic studies that people with advanced age and pre-existing disorders such as extreme liver and kidney diseases are at greater risk (Shen et al., 2020). Furthermore, metabolic biomarkers can also predict heart failure re-hospitalization and mortality (Bader et al., 2020; Chen et al., 2020b).

Various metabolites were observed in COVID-19 patients with unequal abundance, and the severity the of disease can be known by the proteomic and metabolomic profiling of sera. Kynurenate, kynurenine, 8-methoxykynurenate are the enriched metabolites detected in COVID-19 patients. The molecular changes in COVID-19 patient's sinvolvelves macrophage dysregulation, platelet degranulation, and complement system pathways, and major metabolic suppression (Udugama et al., 2020). The major metabolites such as malic acid (Wan et al., 2020), XU-5-P (Wang et al., 2020), Carbamoyl phosphate (Di et al., 2020), Dihydrouracl (Wu et al., 2020), and Glycerol 3-phosphate (Yang et al., 2020) show a reduction in both severe and mild group patients.

\section{Application of Nano-Biotechnology in COIVD-19 Diagnosis}

Nanoparticles (NPs) such as Iron oxide $\left(\mathrm{Fe}_{3} \mathrm{O}_{4}\right)$, gold nanoparticles (AuNPs), Graphene oxide (GO), Quantum dots (QDs), gained considerable attention due to their superparamagnetic, strong magnetic dipole-dipole attraction, and ferromagnetic behavior (Gonzalez-Rodriguez et al., 2019). NPs are used in the development of biosensors, drug delivery, medical diagnostic tools, detection, and separation of pathogens (Zhou et al., 2020). As they are highly sensitive, precise, selective, and cost-effective they are used in the detection of various molecules such as the proteins, DNAs, RNAs, etc., (Campos et al., 2020).

In rGO-SELEX, the reduced GONPs interacts with the nucleotides and helps in the selection process. Whereas, the capture-SELEX involves immobilization of the nucleotide sequences leading to the specific selection of the ssDNA against the target (Zou et al., 2019). The immuno-SELEX includes the preparation of the anti-target antibodies and the use of sandwich ELISA principle for better efficiency. Highly QDs, have a wide range of applications in various diagnostics platforms. However, FRET-based nanosensors conjugated with NPs enable detection of different target small molecules, pathogens, and $\mathrm{H} 5 \mathrm{~N} 1$ avian influenza virus (Bhalla et al., 2020). In addition, AuNPs unique properties functionalized with oligonucleotides (ONT-AuNPs) have emerged as a new kind of novel nanomaterial for diagnosis and therapy by taking advantage of the highly efficient fluorescence quenching properties (Zhang et al., 2020).

\section{SUMMARY AND FUTURE OUTLOOK}

This review provides insights into current diagnostic tools for novel coronavirus (SARS-CoV-2) pandemic in terms of public health impact and emergencies. The detection of nucleic acid by RT-PCR is a gold standard for the diagnosis of the SARS-CoV-2 virus. The typical step in this diagnosis is the extraction of the $\mathrm{RNA}$, and the entire process may take 3-4 $\mathrm{h}$ and requires expensive laboratory facilities and qualified technicians. The RT-PCR diagnostic approach provided the first line of defense against the outbreak. However, various serological diagnostic methods are also available to complement the RT-PCR, but cross-reactivity is an issue, and these methods may result in false positives, which is a major concern. The disease is spreading day by day; thus, the prerequisites at this time are the more affordable Point of Care tools. The aptamer is a novel, short sequence of the oligonucleotide which specifically binds to the target protein. Aptamers are used, in a variety of multiplex fluorescence assays such as FRET, SPR, Raman spectroscopy; proteome assay, chemiluminescence, and lateral flow-based diagnosis which are inexpensive, fast and 
responsive. Hence, they are one of the ideal candidates to focus on the development of PoC devices. There is an urgent need for more diagnostic tools as there is rapid growth in the number of cases worldwide until effective drugs/vaccines reach the market. Detailed studies are required to understand the transmission, host interaction, mutations for the development of effective vaccination to defeat the disease. The SARS-CoV-2 outbreak is an unprecedented disaster in all parts of the world in all aspects, including health and economics. This review would help in the development of new knowledge for the researches working in the diagnostics platforms by using nanobiotechnology based approaches to combat the SARS-CoV-2 virus.

\section{REFERENCES}

Abbott (2020). Abbott ID NOW ${ }^{\mathrm{TM}}$ COVID-19. Available at: https://www.alere. com/en/home/product-details/id-now-covid-19.html

Adamczyk, M., Mattingly, P. G., Shreder, K., and Yu, Z. (1999). Surface plasmon resonance (SPR) as a tool for antibody conjugate analysis. Bioconjugate Chem. 10, 1032-1037. doi:10.1021/bc990057e

Ahn, D. G., Jeon, I. J., Kim, J. D., Song, M. S., Han, S. R., Lee, S. W., et al. (2009). RNA aptamer-based sensitive detection of SARS coronavirus nucleocapsid protein. Analyst. 134 (9), 1896-1901. doi:10.1039/b906788d

Bader, F., Manla, Y., Atallah, B., and Starling, R. C. (2020). Heart failure and COVID-19. Heart Fail. Rev. 21 (1), 1-10. doi:10.1007/s10741-020-10008-2

Bai, C., Lu, Z., Jiang, H., Yang, Z., Liu, X., Ding, H., et al. (2018). Aptamer selection and application in multivalent binding-based electrical impedance detection of inactivated H1N1 virus. Biosens. Bioelectron. 110, 162-167. doi:10.1016/j.bios. 2018.03.047

Bhalla, N., Pan, Y., Yang, Z., and Payam, A. F. (2020). Opportunities and challenges for biosensors and nanoscale analytical tools for pandemics: covid-19. ACS Nano. 14 (7), 7783-7807. doi:10.1021/acsnano.0c04421

Burrel, S., Hausfater, P., Dres, M., Pourcher, V., Luyt, C. E., Teyssou, E., et al. (2020). Co-infection of SARS-CoV-2 with other respiratory viruses and performance of lower respiratory tract samples for the diagnosis of covid19. Int. J. Infect. Dis. 102, 10-13. doi:10.1016/j.ijid.2020.10.040

Bwire, G. M. (2020). Coronavirus: why men are more vulnerable to covid-19 than women? SN Compr. Clin. Med. 2 (7), 874-876. doi:10.1007/s42399-02000341-w

Cai, X. F., Chen, J., Li Hu, J., Long, Q. X., Deng, H. J., Liu, P., et al. (2020). A peptide-based magnetic chemiluminescence enzyme immunoassay for serological diagnosis of coronavirus disease 2019. J. Infect. Dis. 222 (2), 189-193. doi:10.1093/infdis/jiaa243

Campos, E. V. R., Pereira, A. E. S., De Oliveira, J. L., Carvalho, L. B., GuilgerCasagrande, M., De Lima, R., et al. (2020). How can nanotechnology help to combat COVID-19? Opportunities and urgent need. J. Nanobiotechnol. 18, 125. doi:10.1186/s12951-020-00685-4

Cascella, M., Rajnik, M., Cuomo, A., Dulebohn, S. C., and Di Napoli, R. (2020). "Features, evaluation, and treatment of coronavirus," in StatPearls. (Treasure Island, FL: StatPearls Publishing. ). Available at: https://www.ncbi.nlm.nih.gov/ books/NBK554776

Chen, T., Wu, D., Chen, H., Yan, W., Yang, D., Chen, G., et al. (2020b). Clinical characteristics of 113 deceased patients with coronavirus disease 2019: retrospective study. BMJ. 368, m1091. doi:10.1136/bmj.m1091

Chen, Y., Liu, Q., and Guo, D. (2020c). Emerging coronaviruses: genome structure, replication, and pathogenesis. J. Med. Virol. 92 (10), 2249. doi:10.1002/jmv. 2623410.1002/jmv.26234

Chen, Z., Wu, Q., Chen, J., Ni, X., and Dai, J. (2020a). A DNA aptamer based method for detection of SARS-CoV-2 nucleocapsid protein. Virol. Sin. 35 (3), 351-354. doi:10.1007/s12250-020-00236-z

de Carvalho, L., and Nogueira, M. S. (2020). Optical techniques for fast screening towards prevention of the coronavirus COVID-19 outbreak. Photodiagnosis Photodyn. Ther. 30, 101765. doi:10.1016/j.pdpdt.2020.101765

\section{AUTHOR CONTRIBUTIONS}

The author confirms being the sole contributor of this work and has approved it for publication.

\section{ACKNOWLEDGMENTS}

We acknowledge the support of the Department of Biotechnology, Ministry of Science and Technology, India, Council of Scientific and Industrial Research (CSIR), New Delhi, India, and CSIRNational Chemical Laboratory, Pune, India for providing necessary facilities.

Deckert, V., Deckert-Gaudig, T., Cialla-May, D., Popp, J., Zell, R., DeinhardEmmer, S., et al. (2020). Laser spectroscopic technique for direct identification of a single virus I: faster cars. Proc. Natl. Acad. Sci. U.S.A. 117 (45), 27820-27824. doi:10.1073/pnas.2013169117

Di, W., Ting, S., Xiaobo, Y., Jian Xin, S., Mingliang, Z., Chengye, Y., et al. (2020). Plasma metabolomic and lipidomic alterations associated with COVID-19. Nat. Sci. Rev. 7 (7), 1157-1168. doi:10.1093/nsr/nwaa086

El-Tholoth, M., Bau, H. H., and Song, J. Z. (2020). A single, and two-stage, closedtube, molecular test for the 2019 novel coronavirus (COVID-19) at home, clinic, and points of entry. ChemRxiv. doi:10.26434/chemrxiv.11860137.v1

Esbin, M. N., Whitney, O. N., Chong, S., Maurer, A., Darzacq, X., and Tjian, R. (2020). Overcoming the bottleneck to widespread testing: a rapid review of nucleic acid testing approaches for COVID-19 detection. RNA. 26 (7), 771-783. doi:10.1261/rna.076232.120

Fouchier, R. A., Hartwig, N., Bestebroe, T. M., Niemeyer, B., de Jong, J. C., Simon, J. H., et al. (2004). A previously undescribed coronavirus associated with respiratory disease in humans. Proc. Natl. Acad. Sci. USA 101 (16), 6212-6216. doi:10.1073/pnas.0400762101

Fraser, D. D., Slessarev, M., Martin, C. M., Daley, M., Patel, M. A., Miller, M. R., et al. (2020). Metabolomics profiling of critically ill coronavirus disease 2019 patients: identification of diagnostic and prognostic biomarkers. Crit. Care Explor. 2 (10), e0272. doi:10.1097/CCE.0000000000000272

Gonzalez-Rodriguez, R., Campbell, E., and Naumov, A. (2019). Multifunctional graphene oxide/iron oxide nanoparticles for magnetic targeted drug delivery dual magnetic resonance/fluorescence imaging and cancer sensing. PloS one 14 (6), e0217072. doi:10.1371/journal.pone.0217072

Ha, C., Jang, S., Shin, G., Jung, G. Y., and Lee, J. W. (2020). Sensitive one-step isothermal detection of pathogen-derived RNAs. MedRxiv. doi:10.1101/2020. 03.05.20031971

Hilgenfeld, R., and Peiris, M. (2013). From SARS to MERS: 10 years of research on highly pathogenic human coronaviruses. Antivir. Res. 100 (1), 286-295. doi:10. 1016/j.antiviral.2013.08.015

Ihling, C., Tänzler, D., Hagemann, S., Kehlen, A., Hüttelmaier, S., Arlt, C., et al. (2020). Mass spectrometric identification of SARS-CoV-2 proteins from gargle solution samples of COVID-19 patients. J. Proteome Res. 19 (11), 4389-4392. doi:10.1021/acs.jproteome.0c00280

Jacobs, B., Snoeren, N., Samim, M., Rosing, H., de Vries, N., Deenen, M. J., et al. (2018). The impact of liver resection on the dihydrouracil: uracil plasma ratio in patients with colorectal liver metastases. Eur. J. Clin. Pharmacol. 74, 737-744. doi:10.1007/s00228-018-2426-4

Jiang, H. W., Li, Y., Zhang, H. N., Wang, W., Yang, X., Qi, H., et al. (2020). SARSCoV-2 proteome microarray for global profiling of COVID-19 specific IgG and IgM responses. Nat. Commun. 11 (1), 3581. doi:10.1038/s41467-020-17488-8

Jin, J. M., Bai, P., He, W., Wu, F., Liu, X. F., Han, D. M., et al. (2020). Gender differences in patients with COVID-19: focus on severity and mortality. Front. Public Health 8, 152. doi:10.1101/2020.02.23.20026864

Johnson, N. P., and Mueller, J. (2002). Updating the accounts: global mortality of the 1918-1920 "Spanish" influenza pandemic. Bull. Hist. Med. 76 (1), 105-115. doi:10.1353/bhm.2002.0022

Kim, Y., Yaseen, A. B., Kishi, J. Y., Hong, F., Saka, S. K., Sheng, K., et al. (2020). Single-strand RPA for rapid and sensitive detection of SARS-CoV-2 RNA. 
medRxiv. 2020 [The Preprint Server for Health Sciences]. doi:10.1101/2020.08. 17.20177006

Korber, B., Fischer, W. M., Gnanakaran, S., Yoon, H., Theiler, J., Abfalterer, W., et al. (2020). Tracking changes in SARS-CoV-2 spike: evidence that D614G increases infectivity of the COVID-19 virus. Cell 182 (4), 812-827. doi:10.1016/ j.cell.2020.06.043

Kumar, A. R., Mudili, V., and Poda, S. (2018). Development of a FRET-based fluorescence aptasensor for the detection of aflatoxin B1 in contaminated food grain samples. RSC $A d v .8$ (19), 10465-10473. doi:10.1039/c8ra00317c

Lauer, S. A., Grantz, K. H., Bi, Q., Jones, F. K., Zheng, Q., Meredith, H. R., et al. (2020). The incubation period of coronavirus disease 2019 (COVID-19) from publicly reported confirmed cases: estimation and application. Ann. Intern. Med. 172 (9), 577-582. doi:10.7326/M20-0504

Lawrence, C. M., Menon, S., Eilers, B. J., Bothner, B., Khayat, R., Douglas, T., et al. (2009). Structural and functional studies of archaeal viruses. J. Biol. Chem. 284, 12599-12603. doi:10.1074/jbc.R800078200

Lee, J., Jung, J., Ko, T., Kim, S., Kim, S. I., Nah, J., et al. (2017). Catalytic synergy effect of MoS2/reduced graphene oxide hybrids for a highly efficient hydrogen evolution reaction. RSC Adv. 7 (9), 5480-5487. doi:10.1039/c6ra26149c

Lee, S. H. (2020). Testing for SARS-CoV-2 in cellular components by routine nested RT-PCR followed by DNA sequencing. Int. J. Geria. Rehab. 2 (1), 69-96

Letko, M., Marzi, A., and Munster, V. (2020). Functional assessment of cell entry and receptor usage for SARS-CoV-2 and other lineage B betacoronaviruses. Nat Microbiol. 5 (4), 562-569. doi:10.1038/s41564-020-0688-y

Loeffelholz, M. J., and Tang, Y. W. (2020). Laboratory diagnosis of emerging human coronavirus infections-the state of the art. Emerg. Microb. Infect. 9 (1), 747-756. doi:10.1080/22221751.2020.1745095

Lu, R., Zhao, X., Li, J., Niu, P., Yang, B., Wu, H., et al. (2020). Genomic characterization and epidemiology of 2019 novel coronavirus: implications for virus origins and receptor binding. Lancet 395, 565-574. doi:10.1016/S01406736(20)30251-8

Malik, Y. A. (2020). Properties of coronavirus and SARS-CoV-2. Malays. J. Pathol. $42(1), 3-11$

Neuman, B. W., Joseph, J. S., Saikatendu, K. S., Serrano, P., Chatterjee, A., Johnson, M. A., et al. (2008). Proteomics analysis unravels the functional repertoire of coronavirus nonstructural protein 3. J. Virol. 82, 5279-5294. doi:10.1128/JVI. 02631-07

Nikolaev, E. N., Indeykina, M. I., Brzhozovskiy, A. G., Bugrova, A. E., Kononikhin, A. S., Starodubtseva, N. L., et al. (2020). Mass-spectrometric detection of SARS$\mathrm{CoV}-2$ virus in scrapings of the epithelium of the nasopharynx of infected patients via nucleocapsid N protein. J. Proteome Res. 19 (11), 4393-4397. doi:10. 1021/acs.jproteome.0c00412

Rahim Ruslinda, A., Tanabe, K., Ibori, S., Wang, X., and Kawarada, H. (2013). Effects of diamond-FET-based RNA aptamer sensing for detection of real sample of HIV-1 Tat protein. Biosens. Bioelectron. 40 (1), 277-282. doi:10.1016/ j.bios.2012.07.048

Rapid Microbiology (2020). https://www.rapidmicrobiology.com/news/pinpoint39slow-cost-handheld-covid-19-aptamer-based-diagnostic-device-in-development.

Ren, L. L., Wang, Y. M., Wu, Z. Q., Xiang, Z. C., Guo, L., Xu, T., et al. (2020). Identification of a novel coronavirus causing severe pneumonia in humans. Chin. Med. J. 133 (9), 1015-1024. doi:10.1097/CM9.0000000000000722

Rocca, M. F., Zintgraff, J. C., Dattero, M. E., Santos, L. S., Ledesma, M., Vay, C., et al. (2020). A combined approach of MALDI-TOF mass spectrometry and multivariate analysis as a potential tool for the detection of SARS-CoV-2 virus in nasopharyngeal swabs. J. Virol. Methods. 286, 113991. doi:10.1016/j.jviromet. 2020.113991

Rosenwald, M. S. (2020). History's deadliest pandemics, from ancient Rome to modern America. The Washington Post, April, 7.

Ryan, K. J., and Ray, C. G. (2004). Sherris medical microbiology. 4th Edn (New York, NY: McGraw-Hill), 525-528.

Samson, R., Navale, G. R., and Dharne, M. S. (2020). Biosensors: frontiers in rapid detection of COVID-19. 3 Biotech. 10 (9), 385. doi:10.1007/s13205-02002369-0

Sergei, A. K., Kurseev, A. M., Solovjev, M. M., Neumann, M. M., Medvedko, A. V., and Sakharov, I. Y. (2020). Chemiluminescent and colorimetric aptamer-based assays of human a-thrombin. Anal. Lett. 53, 140-151. doi:10.1080/00032719. 2019.1640718

Shafiei, F., McAuliffe, K., Bagheri, Y., Sun, Z., Yu, Q., Wu, R., et al. (2020). Paperbased fluorogenic RNA aptamer sensors for label-free detection of small molecules. Anal. Methods : Advancing Methods and Applications. 12 (21), 2674-2681. doi:10.1039/d0ay00588f

Shanmugaraj, B., Siriwattananon, K., Wangkanont, K., and Phoolcharoen, W. (2020). Perspectives on monoclonal antibody therapy as potential therapeutic intervention for Coronavirus disease-19 (COVID-19). Asian Pac. J. Allergy Immunol. 38 (1), 10-18. doi:10.12932/AP-200220-0773

Shen, B., Yi, X., Sun, Y., Bi, X., Du, J., Zhang, C., et al. (2020). Proteomic and metabolomic characterization of COVID-19 patient sera. MedRxiv, 4585. doi:10.1101/2020.04.07.20054585

Smyrlaki, I., Ekman, M., Lentini, A., de Sousa, N. R., Papanicolaou, N., Vondracek, M., et al. (2020). Massive and rapid COVID-19 testing is feasible by extraction-free SARSCoV-2 RT-PCR. Nat. Commun. 11 (1), 4812. doi:10.1038/s41467-020-18611-5

Tsang, K. W., Ho, P. L., Ooi, G. C., Yee, W. K., Wang, T., Chan-Yeung, M., et al. (2013). A cluster of cases of severe acute respiratory syndrome in Hong Kong. N. Engl. J. Med. 348 (20), 1977-1985. doi:10.1056/NEJMoa030666

Udugama, B., Kadhiresan, P., Kozlowski, H. N., Malekjahani, A., Osborne, M., Li, V. Y. C., et al. (2020). Diagnosing COVID-19: the disease and tools for detection. ACS Nano. 14, 3822-3835. doi:10.1021/acsnano.0c02624

Virus Taxonomy (2019). Virus Taxonomy Release. talk.ictvonline.org. International committee on Taxonomy of viruses. April, 252020.

Wan, Y., Shang, J., Graham, R., Baric, R. S., and Li, F. (2020). Receptor recognition by a novel coronavirus from Wuhan: an analysis based on decade-long structural studies of SARS. J. Virol. 94 (7), 120-127. doi:10.1128/jvi.00127-2

Wang, H., Hou, X., Wu, X. L. T., Hou, X., Liang, T., Wang, D., et al. (2020). SARSCoV-2 proteome microarray for mapping COVID-19 antibody interactions at amino acid resolution. BioRxiv. 6 (12), 2238-2249. doi:10.1101/2020.03.26.994756

Woo, C. H., Jang, S., Shin, G., Jung, G. Y., and Lee, J. W. (2020). Sensitive fluorescence detection of SARS-CoV-2 RNA in clinical samples via one-pot isothermal ligation and transcription. Nat Biomed Eng. 4, 1168-1179. doi:10. 1038/s41551-020-00617-5

Woo, P. C., Lau, S. K., Chu, C. M., Chan, K. H., Tsoi, H. W., Huang, Y., et al. (2005). Characterization and complete genome sequence of a novel coronavirus, coronavirus HKU1, from patients with pneumonia. J. Virol. 79 (2), 884-895. doi:10.1128/JVI.79.2.884-895.2005

Wu, D., Shu, T., Yang, X., Song, J.-X., Zhang, M., Yao, C., et al. (2020). Plasma metabolomic and lipidomic alterations associated with COVID-19. Nat. Sci. Rev. 7, 1157-1168. doi:10.1093/nsr/nwaa086

Yang, T., Wang, Y. C., Shen, C. F., and Cheng, C. M. (2020). Point-of-care RNAbased diagnostic device for COVID-19. Diagnostics. 10, 165. doi:10.3390/ diagnostics10030165

Zhang, J., Mou, L., and Jiang, X. (2020). Surface chemistry of gold nanoparticles for health-related applications. Chem. Sci. 11 (4), 923-936. doi:10.1039/c9sc06497d

Zhou, P., Yang, X. L., Wang, X. G., Hu, B., Zhang, L., Zhang, W., et al. (2020). A pneumonia outbreak associated with a new coronavirus of probable bat origin. Nature. 579, 270-273. doi:10.1038/s41586-020-2012-7

Zon, G. (2020). Recent advances in aptamer applications for analytical biochemistry. Anal. Biochem. 2020, 113894. doi:10.1016/j.ab.2020.113894

Zou, X., Wu, J., Gu, J., Shen, L., and Mao, L. (2019). Application of aptamers in virus detection and antiviral therapy. Front. Microbiol. 10, 1462. doi:10.3389/ fmicb.2019.01462

Conflict of Interest: The author declares that the research was conducted in the absence of any commercial or financial relationships that could be construed as a potential conflict of interest.

Copyright (c) 2021 Koteswara Rao. This is an open-access article distributed under the terms of the Creative Commons Attribution License (CC BY). The use, distribution or reproduction in other forums is permitted, provided the original author(s) and the copyright owner(s) are credited and that the original publication in this journal is cited, in accordance with accepted academic practice. No use, distribution or reproduction is permitted which does not comply with these terms. 